\title{
Controllability Metrics, Limitations and Algorithms for Complex Networks
}

\author{
Fabio Pasqualetti, Sandro Zampieri, and Francesco Bullo
}

\begin{abstract}
This paper studies the problem of controlling stable and symmetric complex networks, that is, the joint problem of selecting a set of control nodes and of designing a control input to drive a network to a target state. We adopt the smallest eigenvalue of the controllability Gramian as metric for the controllability degree of a network, as it identifies the energy needed to accomplish the control task. In the first part of the paper we characterize tradeoffs between the control energy and the number of control nodes, based on the network topology and weights. Our bounds show for instance that, if the number of control nodes is constant, then the control energy increases exponentially with the number of network nodes. Consequently, despite the classic controllability notion, few nodes cannot in practice arbitrarily symmetric control complex networks. In the second part of the paper we propose a distributed openloop strategy with performance guarantees for the control of complex networks. In our strategy we select control nodes based on network partitioning, and we design the control input based on optimal and distributed control techniques. For our control strategy we show that the control energy depends on the controllability properties of the clusters and on their coupling strength, and it is independent of the network dimension.
\end{abstract}

\section{INTRODUCTION}

The ability to control complex networks via external inputs is fundamental to guarantee reliable and efficient network operation. Despite important advances in control theory, several questions regarding the control of complex networks are largely unexplored, as, for instance, the relation between network topology and its controllability degree.

The control problem of complex networks consists of the selection of a set of control nodes, and the design of a control law to steer the network to a target state. We focus on stable and symmetric networks, and we refer the reader to [1] for the case of unstable and asymmetric networks. We adopt the worst-case energy to drive a network from the origin to a target state as controllability metric. By combining this controllability notion with graph theory, we characterize tradeoffs between the energy to control a given network and the number of control nodes, and we develop a distributed control strategy with guaranteed performance and computational complexity.

Related work The classic concept of controllability has found renewed interest in the context of complex networks,

This material is based upon work supported in part by NSF Award 1135819 and in part by ARO Award W911NF-11-1-0092 and by European Communitys Seventh Framework Program under grant agreement n. 257462 HYCON2 Network of Excellence. Fabio Pasqualetti is with the Mechanical Engineering Department, University of California at Riverside, fabiopaseengr.ucr.edu. Sandro Zampieri is with the Department of Information Engineering, University of Padova, Italy, sandro.zampieri@dei.unipd.it. Francesco Bullo is with the Mechanical Engineering Department and the Center for Control, Dynamical Systems and Computation, University of California at Santa Barbara, bullo@engineering.ucsb.edu. where classic methods are often inapplicable due to the system dimension, and where a graph-inspired understanding of controllability rather than a matrix-theoretical one is preferable. For instance, in [2] standard tools from structured control theory [3] are used to investigate controllability properties of real networks. Among other conclusions, the analysis in [2] reveals that the required number of control nodes is mainly related to the network degree distribution, and that sparse inhomogeneous networks are most difficult to control, while dense and homogeneous networks require only a few control nodes. The approach to controllability undertaken in [2] has several shortcomings. First, the presented results are generic, in the sense that they hold for almost every choice of the network parameters [4], but they may fail to hold if certain symmetries or constraints are present [3, Section 15], [5]. Second, most results in [2], [6] rely on particular interconnection properties of the considered networks, perhaps the absence of self-loops around the network nodes. In fact it follows from [3, Theorem 14.2], equivalently from [7, Theorem 1], that every strongly connected network with self-loops is generically controllable by any single node, which contradicts the conclusions drawn in [2]. This discrepancy is underlined in [8] for the case of biological networks, and more generally in [9]. Third, the binary notion of controllability proposed in [10] and adopted in [2] does not characterize the difficulty of the control task. In practice, although a network may be generically controllable by any single node, the actual control input may not be implementable due to actuator constraints and limitations. Finally, the design of the control input to drive a network to a particular state is not specified in [2], and it remains to date an outstanding problem for complex networks due to their dimension and absence of a central controller.

We depart from [2], [6], [5], [9], and analogously from [11], [12], [13], by adopting a quantitative measure of network controllability, namely the worst-case control energy, by characterizing tradeoffs between the difficulty of the control task and the number of control nodes, and by proposing an open-loop control strategy for complex networks.

Contribution This paper features three main contributions.

First, we study network controllability from an energy perspective, which we quantify with the smallest eigenvalue of the controllability Gramian (Section II). We show that certain controllable networks are practically uncontrollable by few control nodes, as the control energy grows exponentially in the network cardinality.

Second, we derive an upper bound for the smallest eigenvalue of the controllability Gramian as a function of the number of control nodes (Section III). Our bound shows, 
for instance, that in order to control a network with constant energy, the number of control nodes must grow linearly with the network dimension. These result provides a quantitative measure of the numerical findings in [14], and are in accordance with existing results in control theory [15].

Third and finally, we propose the decoupled control strategy for the control of complex networks (Section IV). The decoupled control strategy consists of network partitioning, selection of the control nodes, and the design of a distributed control law to steer the network to a target state. We characterize the performance of the decoupled control strategy, and we show that, if sufficiently many control nodes are available, the energy to control a network depends only on the controllability properties of its parts, and on their coupling strength. Conversely, we prove that certain networks admit a distributed control strategy where the control energy is independent of the network dimension. To the best of our knowledge, our decoupled control strategy is novel, it constitutes a first solution for the distributed scalable control of complex networks, and it leads to a novel notion of network controllability centrality.

\section{Network MOdel AND Preliminary Results}

Consider a network represented by the undirected graph $\mathcal{G}:=(\mathcal{V}, \mathcal{E})$, where $\mathcal{V}:=\{1, \ldots, n\}$ and $\mathcal{E} \subseteq \mathcal{V} \times \mathcal{V}$ are the vertices and the edges sets, respectively. Let $a_{i j} \in \mathbb{R}$ be the weight associated with the edge $(i, j) \in \mathcal{E}$, and define the weighted adjacency matrix of $\mathcal{G}$ as $A=A^{\top}=\left[a_{i j}\right]$, where $a_{i j}=0$ whenever $(i, j) \notin \mathcal{E}$. We associate a real value (state) with each node, collect the nodes states into a vector (network state), and define the map $x: \mathbb{N}_{\geq 0} \rightarrow \mathbb{R}^{n}$ to describe the evolution (network dynamics) of the network state over time. We consider the discrete time linear and time-invariant network dynamics described by the equation

$$
x(t+1)=A x(t) .
$$

Controllability of the network $\mathcal{G}$ refers to the possibility of steering the network state to an arbitrary configuration by means of external controls. We assume that a set

$$
\mathcal{K}:=\left\{k_{1}, \ldots, k_{m}\right\} \subseteq \mathcal{V}
$$

of nodes can be independently controlled, and we let

$$
B_{\mathcal{K}}:=\left[\begin{array}{lll}
e_{k_{1}} & \cdots & e_{k_{m}}
\end{array}\right]
$$

be the input matrix, where $e_{i}$ denotes the $i$-th canonical vector of dimension $n$. The network with control nodes $\mathcal{K}$ reads as

$$
x(t+1)=A x(t)+B_{\mathcal{K}} u_{\mathcal{K}}(t),
$$

where $u_{\mathcal{K}}: \mathbb{N}_{\geq 0} \rightarrow \mathbb{R}$ is the control signal injected into the network via the nodes $\mathcal{K}$. A network is controllable in $T \in \mathbb{N}$ steps by the set of control nodes $\mathcal{K}$ if and only if for every state $x_{\mathrm{f}} \in \mathbb{R}^{n}$ there exists an input $u_{\mathcal{K}}$ such that $x(T)=x_{\mathrm{f}}$ with $x(0)=0$ [16]. Controllability of dynamical systems is a well-understood property, and it can be checked by different structural conditions [10], [17], [3]. For instance, let $\mathcal{C}_{\mathcal{K}, T}$, with $T \in \mathbb{N}_{\geq 1}$, be the controllability matrix defined as

$$
\mathcal{C}_{\mathcal{K}, T}:=\left[\begin{array}{llll}
B_{\mathcal{K}} & A B_{\mathcal{K}} & \cdots & A^{T-1} B_{\mathcal{K}}
\end{array}\right] .
$$

The network (3) is controllable in $T$ steps by the nodes $\mathcal{K}$ if and only if the controllability matrix $\mathcal{C}_{\mathcal{K}, T}$ is of full row rank.

The above notion of controllability is qualitative, and it does not quantify the difficulty of the control task as measured, for instance, by the control energy needed to reach a desired state. As a matter of fact, many controllable networks require very large control energy to reach certain states [14]. To formalize this discussion, define the $T$-steps controllability Gramian by

$$
\mathcal{W}_{\mathcal{K}, T}:=\sum_{\tau=0}^{T-1} A^{\tau} B_{\mathcal{K}} B_{\mathcal{K}}^{\top} A^{\tau}=\mathcal{C}_{\mathcal{K}, T} \mathcal{C}_{\mathcal{K}, T}^{\top},
$$

where we have used the fact that $A=A^{\top}$. It can be easily verified that the controllability Gramian $\mathcal{W}_{\mathcal{K}, T}$ is positive definite if and only if the network is controllable in $T$ steps by the nodes $\mathcal{K}[16]$.

Let the network be controllable in $T$ steps, and let $x_{\mathrm{f}}$, with $\left\|x_{\mathrm{f}}\right\|=1$, be the desired final state at time $T$. Define the energy of the control input $u_{\mathcal{K}}$ as

$$
\mathrm{E}\left(u_{\mathcal{K}}, T\right):=\left\|u_{\mathcal{K}}\right\|_{2, T}^{2}=\sum_{\tau=0}^{T-1}\left\|u_{\mathcal{K}}(\tau)\right\|_{2}^{2},
$$

where $T$ is the control horizon. The unique control input that steers the network state from $x(0)=0$ to $x(T)=x_{\mathrm{f}}$ with minimum energy is [16]

$$
u_{\mathcal{K}}^{*}(t):=B_{\mathcal{K}}^{\top} A^{T-t-1} \mathcal{W}_{\mathcal{K}, T}^{-1} x_{\mathrm{f}},
$$

with $t \in\{0, \ldots, T-1\}$. Then, it can be seen that

$$
\mathrm{E}\left(u_{\mathcal{K}}^{*}, T\right)=\sum_{\tau=0}^{T-1}\left\|u_{\mathcal{K}}^{*}(\tau)\right\|_{2}^{2}=x_{\mathrm{f}}^{\top} \mathcal{W}_{\mathcal{K}, T}^{-1} x_{\mathrm{f}} \leq \lambda_{\min }^{-1}\left(\mathcal{W}_{\mathcal{K}, T}\right)
$$

where equality is achieved whenever $x_{\mathrm{f}}$ is an eigenvector of $\mathcal{W}_{\mathcal{K}, T}$ associated with $\lambda_{\min }\left(\mathcal{W}_{\mathcal{K}, T}\right)$. Because the control energy is limited in practical applications, controllable networks featuring small Gramian eigenvalues cannot be steered to certain states. An example follows.

Example 1: (Controllable networks may exhibit practically uncontrollable states) Consider the network $\mathcal{G}$ with $n$ nodes, weighted adjacency matrix $A:=\left[a_{i j}\right]$ defined as

$$
a_{i j}:= \begin{cases}\frac{1}{2}, & \text { if } j=i-1 \text { and } i \in\{2, \ldots, n\} \\ 0, & \text { otherwise, }\end{cases}
$$

and control node $\mathcal{K}=\{1\}$. Notice that the controllability matrix $\mathcal{C}_{\mathcal{K}, n}$ is diagonal and nonsingular, and its $i$-th diagonal entry equals $2^{-i+1}$. Since $A^{t} B_{\mathcal{K}}=0$ for all $t \geq n$, we have $\mathcal{W}_{\mathcal{K}, \tau}=\mathcal{C}_{\mathcal{K}, n} \mathcal{C}_{\mathcal{K}, n}^{\top}$ for all $\tau \geq n$, and the smallest eigenvalue of the controllability Gramian $\mathcal{W}_{\mathcal{K}, \tau}$ equals $2^{-2 n+2}$ for all $\tau \geq n$. We conclude that the network $\mathcal{G}$ with control nodes $\mathcal{K}$ is controllable in $T \geq n$ steps, yet the control energy grows exponentially with the network cardinality.

In this work we measure controllability of a network based on the smallest eigenvalue of the controllability Gramian, that is, from a worst-case perspective, by looking at the target states requiring the largest control energy to be reached; see [1] for a discussion of alternative controllability metrics. 


\section{LOWER BOUND ON THE CONTROL ENERGY}

In this section we characterize a tradeoff between the energy to control a network and the number of control nodes. Let $\operatorname{spec}(M)$ denote the set of eigenvalues of $M$, and let

$$
\begin{aligned}
\lambda_{\min }(M) & :=\min \{|\lambda|: \lambda \in \operatorname{spec}(M)\}, \\
\lambda_{\max }(M) & :=\max \{|\lambda|: \lambda \in \operatorname{spec}(M)\} .
\end{aligned}
$$

Recall that a matrix $M$ is Schur stable if $\lambda_{\max }(M)<1$.

Theorem 3.1: (Control energy and number of control nodes) Consider a network $\mathcal{G}=(\mathcal{V}, \mathcal{E})$ with $|\mathcal{V}|=n$, weighted adjacency matrix $A$, and control set $\mathcal{K}$. Assume that $A$ is Schur stable. For all $T \in \mathbb{N}_{>0}$ it holds

$$
\lambda_{\min }\left(\mathcal{W}_{\mathcal{K}, T}\right) \leq \min \left\{\frac{1-\lambda_{\min }^{2 T}(A)}{1-\lambda_{\min }^{2}(A)}, \frac{\lambda_{\max }^{2\left(\left\lceil\frac{n}{|K|}\right\rceil-1\right)}(A)}{1-\lambda_{\max }^{2}(A)}\right\} .
$$

Proof: We start by showing the first part of the inequality. Notice that for all $T \in \mathbb{N}_{>0}$ it holds $\lambda_{\min }\left(\mathcal{W}_{\mathcal{K}, T}\right) \leq$ $\lambda_{\min }\left(\mathcal{W}_{\mathcal{V}, T}\right)$. In fact, $\mathcal{K} \subseteq \mathcal{V}$, and the control energy cannot increase by adding control nodes to a given control set. Then,

$$
\lambda_{\min }\left(\mathcal{W}_{\mathcal{V}, T}\right)=\lambda_{\min }\left(\sum_{\tau=0}^{T-1} A^{2 \tau}\right)=\frac{1-\lambda_{\min }(A)^{2 T}}{1-\lambda_{\min }(A)^{2}},
$$

and the first statement follows. We now show the second part of the inequality. Let $T_{\max }=\left\lceil\frac{n}{|\mathcal{K}|}\right\rceil-1$. Notice that $\operatorname{rank}\left(\mathcal{C}_{\mathcal{K}, T_{\text {max }}}\right)<n$. In fact, $\mathcal{C}_{\mathcal{K}, T_{\max }} \in \mathbb{R}^{n \times m}$ with

$$
m=T_{\max }|\mathcal{K}|<\left(\frac{n}{|\mathcal{K}|}+1\right)|\mathcal{K}|-|\mathcal{K}|=n .
$$

Consequently, $\mathcal{W}_{\mathcal{K}, T_{\max }}=\mathcal{C}_{\mathcal{K}, T_{\max }} \mathcal{C}_{\mathcal{K}, T_{\max }}^{\top}$ is singular, and $\lambda_{\min }\left(\mathcal{W}_{\mathcal{K}, T_{\max }}\right)=0$. Let $T>T_{\max }$. Since $\mathcal{W}_{\mathcal{K}, T}$ is symmetric, it admits an orthonormal basis of eigenvectors, that is, $\mathcal{W}_{\mathcal{K}, T}=V^{-1} \Lambda V$, with $V^{\top} V=I$ and $\|V\|_{2}\left\|V^{-1}\right\|_{2}=1$. From the Bauer-Fike Theorem [18] we have

$$
\max _{\mu \in \sigma\left(W_{\mathcal{K}, T_{\max }}\right.} \min _{\lambda \in \sigma\left(W_{K, T}\right)}|\lambda-\mu| \leq\|V\|_{2}\left\|V^{-1}\right\|_{2}\left\|\Delta_{T_{\max }}\right\|_{2},
$$

where $\Delta_{T_{\max }}=-\sum_{\tau=T_{\max }}^{T-1} A^{\tau} B_{\mathcal{K}} B_{\mathcal{K}}^{\top} A^{\tau}$. In particular, since $\mathcal{W}_{\mathcal{K}, T_{\max }}$ is singular, we have

$$
\lambda_{\min }\left(\mathcal{W}_{K, T}\right) \leq\left\|\Delta_{T_{\max }}\right\|_{2} \leq \frac{\lambda_{\max }^{2 T_{\max }}(A)}{1-\lambda_{\max }^{2}(A)},
$$

and the statement follows.

In Theorem 3.1 we provide an upper bound on the smallest eigenvalue of the controllability Gramian or, equivalently, a lower bound on the worst-case energy needed to control a network to an arbitrary target state, as a function of the number of control nodes. In fact, due to (5),

$$
\max _{x_{\mathrm{f}} \in \mathbb{R}^{n}} \mathrm{E}\left(u_{\mathcal{K}}^{*}, T\right) \geq \max \left\{\frac{1-\lambda_{\min }^{2}(A)}{1-\lambda_{\min }^{2 T}(A)}, \frac{1-\lambda_{\max }^{2}(A)}{\lambda_{\max }^{2\left(\left\lceil\frac{n}{|\mathcal{K}|}\right\rceil-1\right)}(A)}\right\},
$$

where $u_{\mathcal{K}}^{*}$ depends on the target state $x_{\mathrm{f}}$ as in (4). The bounds in (7) are to be regarded as performance limitations: independently of the control strategy, the least amount of

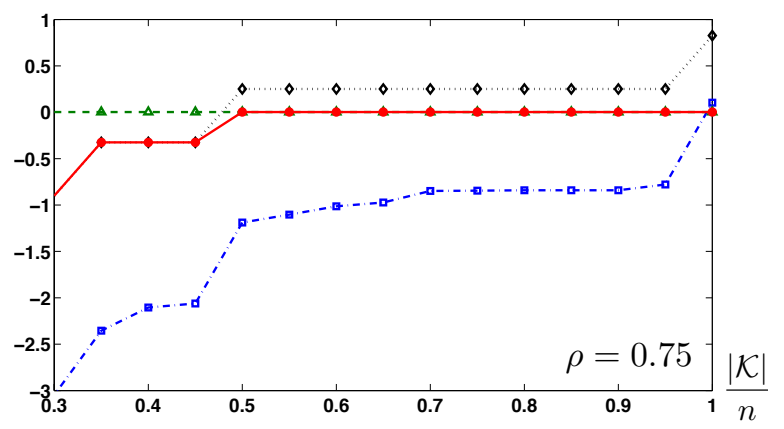

Fig. 1. For the network in Example 2, this figure compares (in a logarithmic scale) the upper bound (7) (solid red) with the largest $\lambda_{\text {min }}^{*}$ of the controllability Gramian (dashed-dot blue) over all possible sets $\mathcal{K}$. For each value $|\mathcal{K}|$ from 1 to $n$, a combinatorial search determines the value $\lambda_{\text {min }}^{*}=\max _{\mathcal{K}} \lambda_{\min }\left(\mathcal{W}_{\mathcal{K}, \infty}\right)$. The two quantities in the right hand side of equation (7) are also reported in dashed green and dotted black, respectively. The horizontal axis represents the ratio $|\mathcal{K}| / n$. It can be shown that the bound (7) tends to be conservative as the parameter $\rho$ increases.

energy needed to steer the network from the origin to an arbitrary unit-norm state is bounded by (7).

Theorem 3.1 implies that, for the control energy to be bounded as the network grows, the number of control nodes must be a linear function of the total number of nodes, provided that $\lambda_{\max }(A)$ remains bounded away from 1 . Instead, classic controllability [2], [10] is (generically) ensured by the presence of a single control node, independently of the network dimension [3, Theorem 14.2], [7, Theorem 1].

Example 2: (Tightness of the bound in Theorem 3.1) Consider a network with $n=20$ nodes and adjacency matrix

$$
A:=\frac{\rho}{3}\left[\begin{array}{ccccc}
1 & 1 & 0 & \cdots & 1 \\
1 & 1 & 1 & \cdots & 0 \\
\vdots & \ddots & \ddots & \ddots & \vdots \\
0 & \cdots & 1 & 1 & 1 \\
1 & \cdots & 0 & 1 & 1
\end{array}\right],
$$

where $\rho \in(0,1)$. Observe that $A$ is Schur stable for all $\rho \in$ $(0,1)$. In Fig. 1 we compare the upper bound in Theorem 3.1 with the value $\max \left\{\lambda_{\min }\left(\mathcal{W}_{\mathcal{K}, T}\right): \mathcal{K} \subseteq\{1, \ldots, 12\},|\mathcal{K}|=\right.$ $k\}$, as a function of the cardinality $k$ of the control set.

\section{Decoupled Control of Complex Networks}

In this section we provide a solution to the problem of controlling a complex network, that is, the problems of both selecting the control nodes, and designing a distributed control law to drive the network to a target state. Our approach is different from classic solutions, as it exploit the network structure to jointly select the control nodes and to design a control law amenable to distributed implementation.

\section{A. Setup and definition of the decoupled control strategy}

Our decoupled control strategy can be divided into three parts: (i) network partitioning, (ii) selection of the control nodes, and (iii) definition of the decoupled control law.

Network partitioning Consider an undirected network $\mathcal{G}:=$ $(\mathcal{V}, \mathcal{E})$ with weighted (symmetric) adjacency matrix $A:=$ $\left[a_{i j}\right]$. Partition $\mathcal{V}$ into $N$ disjoint sets $\mathcal{P}:=\left\{\mathcal{V}_{1}, \ldots, \mathcal{V}_{N}\right\}$, 
and let $\mathcal{G}_{i}:=\left(\mathcal{V}_{i}, \mathcal{E}_{i}\right)$ be the $i$-th subgraph of $\mathcal{G}$ with vertices $\mathcal{V}_{i}$ and edges $\mathcal{E}_{i}:=\mathcal{E} \cap\left(\mathcal{V}_{i} \times \mathcal{V}_{i}\right)$. ${ }^{1}$ According to this partition, and possibly after relabeling states and inputs, the network matrices read as

$$
A=\left[\begin{array}{ccc}
A_{1} & \cdots & A_{1 N} \\
\vdots & \vdots & \vdots \\
A_{N 1} & \cdots & A_{N}
\end{array}\right], B_{\mathcal{K}}=\left[\begin{array}{ccc}
B_{\mathcal{K}_{1}} & \cdots & 0 \\
\vdots & \ddots & \vdots \\
0 & \cdots & B_{\mathcal{K}_{N}}
\end{array}\right]
$$

where $\mathcal{K}_{i} \subseteq \mathcal{V}_{i}$ for all $i \in\{1, \ldots, N\}$, and the networks dynamics can be written as the interconnection of $N$ subsystems of the form

$$
x_{i}(t+1)=A_{i} x_{i}(t)+\sum_{j \in \mathcal{N}_{i}} A_{i j} x_{j}(t)+B_{\mathcal{K}_{i}} u_{\mathcal{K}_{i}}(t),
$$

where $i \in\{1, \ldots, N\}$ and $\mathcal{N}_{i}:=\left\{j: A_{i j} \neq 0\right\}$.

Selection of the control nodes For a network $\mathcal{G}:=(\mathcal{V}, \mathcal{E})$ with partition $\mathcal{P}:=\left\{\mathcal{V}_{1}, \ldots, \mathcal{V}_{N}\right\}$, we say that a node $i \in \mathcal{V}_{k}$ is a boundary node if $a_{i j} \neq 0$ for some node $j \in \mathcal{V}_{\ell}$, with $k, \ell \in\{1, \ldots, N\}$ and $k \neq \ell$. Let $\mathcal{B}_{i} \subseteq \mathcal{V}_{i}$ be the set of boundary nodes in the $i$-th cluster, and let $\mathcal{B}=\bigcup_{i=1}^{N} \mathcal{B}_{i}$ be the set of all the boundary nodes of the partition. We select the set of control nodes $\mathcal{K}=\mathcal{K}_{1} \cup \cdots \cup \mathcal{K}_{N}$ to satisfy $\mathcal{B}_{i} \subseteq \mathcal{K}_{i} \subseteq \mathcal{V}_{i}$ for all $i \in\{1, \ldots, N\}$, and to ensure that each pair $\left(A_{i}, B_{i}\right)$ is controllable. See Fig. 2 for an example.

The decoupled control law For a network $\mathcal{G}:=(\mathcal{V}, \mathcal{E})$ with partition $\mathcal{P}:=\left\{\mathcal{V}_{1}, \ldots, \mathcal{V}_{N}\right\}$, let $x_{\mathrm{f}}^{\top}:=\left[\begin{array}{lll}x_{\mathrm{f} 1}^{\top} & \cdots & x_{\mathrm{f} N}^{\top}\end{array}\right]$ be the target state, where $\left\|x_{\mathrm{f}}\right\|_{2}=1$, and $x_{\mathrm{f} i} \in \mathbb{R}^{\left|\mathcal{V}_{i}\right|}$ for $i \in$ $\{1, \ldots, N\}$. Let $\left\|x_{\mathrm{f}, i}\right\|_{2}=\alpha_{i}$, and notice that $\sum_{i=1}^{N} \alpha_{i}^{2}=1$. Define the control input $u_{\mathcal{K}_{i}}$ by

$$
u_{\mathcal{K}_{i}}(t):=\underbrace{B_{\mathcal{K}_{i}}^{\top} A_{i}^{T-t-1} \mathcal{W}_{i, T}^{-1} x_{\mathrm{f} i}}_{v_{i}(t)}-\sum_{j \in \mathcal{N}_{i}} \underbrace{B_{\mathcal{K}_{i}}^{\top} A_{i j} x_{j}(t)}_{f_{i j}(t)},
$$

where, with a slight abuse of notation, $\mathcal{W}_{i, T}$ is the $i$-th controllability Gramian defined by

$$
\mathcal{W}_{i, T}:=\sum_{\tau=0}^{T-1} A_{i}^{T-\tau-1} B_{\mathcal{K}_{i}} B_{\mathcal{K}_{i}}^{\top} A_{i}^{T-\tau-1},
$$

and the control horizon $T$ is chosen large enough so that $\mathcal{W}_{i, T}$ is positive definite for all $i \in\{1, \ldots, N\}$. We refer to the above control law as to the decoupled control law.

Before analyzing the performance of our decoupled control law, we discuss its implementation properties. First, notice that the control input $u_{\mathcal{K}_{i}}$ is the sum of an open-loop control signal $v_{i}$, and a feedback control signal $\sum_{j \in \mathcal{N}_{i}} f_{i j}$. Second, if each cluster is equipped with a control center, then our decoupled control law can be implemented via distributed computation by the control centers. In fact, the control signal $v_{i}$ depends on the dynamics of only the $i$-th cluster, and the feedback control signals $f_{i j}$ can be determined upon communication of the $i$-th control center with its neighboring control centers belonging to $\mathcal{N}_{i}$. Third

\footnotetext{
${ }^{1}$ Several methods are available to partition a network [19], such as spectral methods and modularity based heuristics. For the implementation of our decoupled control law it is only required that the network is partitioned into strongly connected components. The performance of the decoupled control law depend on the partitioning scheme.
}

and finally, our decoupled control law is scalable, in the sense that the complexity of the control law does not depend upon the network cardinality, but only on its partition, provided that the degree of each cluster remains bounded.

\section{B. Analysis of the decoupled control law}

We start our analysis by noticing that the decoupled control law (10) steers the network to the target state $x_{\mathrm{f}}$. In fact, from equation (9) and the definition of $f_{i j}$ in equation (10), the network dynamics with decoupled control law can be written as the collection of $N$ decoupled subsystems

$$
x_{i}(t+1)=A_{i} x_{i}(t)+B_{\mathcal{K}_{i}} v_{i}(t), i \in\{1, \ldots, N\} .
$$

Since $v_{i}$ in equation (10) equals the minimum energy input to drive the $i$-th subsystem (11) from $x_{i}(0)=0$ to $x_{i}(T)=x_{\mathrm{f} i}$, we conclude that $x(T)=x_{\mathrm{f}}$.

We next study the energy properties of our decoupled control law. Observe that the state evolution of the $i$-th cluster can be written as

$$
x_{i}(t)=\sum_{\tau=0}^{t-1} A_{i}^{t-\tau-1} B_{\mathcal{K}_{i}} B_{\mathcal{K}_{i}}^{\top} A_{i}^{T-\tau-1} \mathcal{W}_{i, T}^{-1} x_{\mathrm{f} i}
$$

For the ease of notation we assume that the matrix $A_{i}$ is Schur stable for all $i \in\{1, \ldots, N\}$. Observe that, if $A$ is Schur stable and nonnegative, then each matrix $A_{i}$ is Schur stable and $\lambda_{\max }\left(A_{i}\right) \leq \lambda_{\max }(A)$. We define the local energy matrix $\Lambda \in \mathbb{R}^{N \times N}$ and the $\mathcal{L}_{2}$ gains matrix $\Gamma \in \mathbb{R}^{N \times N}$ by

$$
\begin{aligned}
& \Lambda:=\operatorname{diag}\left(\lambda_{\min }^{-1}\left(\mathcal{W}_{1, T}\right), \ldots, \lambda_{\min }^{-1}\left(\mathcal{W}_{N, T}\right)\right) \\
& \Gamma:=\left[\begin{array}{cccc}
1 & \gamma_{12} & \ldots & \gamma_{1 N} \\
\gamma_{21} & 1 & \ldots & \gamma_{2 N} \\
\vdots & \vdots & \ddots & \vdots \\
\gamma_{N 1} & \gamma_{N 2} & \ddots & 1
\end{array}\right]
\end{aligned}
$$

where $\gamma_{i j}$, for $i, j \in\{1, \ldots, N\}$ and $i \neq j$, is the $\mathcal{L}_{2}$ gain of the system $\left(A_{j}, B_{\mathcal{K}_{j}}, B_{\mathcal{K}_{i}}^{\top} A_{i j}\right)$ or, equivalently, the $H_{\infty}$ gain of the transfer matrix $B_{\mathcal{K}_{i}}^{\top} A_{i j}\left(z I-A_{j}\right)^{-1} B_{\mathcal{K}_{j}}$ [20].

Theorem 4.1: (Energy of the decoupled control law) Consider a network $\mathcal{G}=(\mathcal{V}, \mathcal{E})$ with weighted adjacency matrix $A=A^{\top}$, control set $\mathcal{K}$, and partition $\mathcal{P}$. Assume that $\mathcal{K}$ contains all boundary nodes of $\mathcal{P}$, every $A_{i}$ is stable, and every pair $\left(A_{i}, B_{i}\right)$ is controllable, where $A_{i}$ and $B_{i}$ are the submatrices associated with the partition $\mathcal{P}$. The decoupled control law $u_{\mathcal{K}}^{\mathrm{d}}$ with control horizon $T$ satisfies

$$
\mathrm{E}\left(u_{\mathcal{K}}^{\mathrm{d}}, T\right) \leq\left\|\Gamma \Lambda^{1 / 2}\right\|_{2}^{2},
$$

where $\Lambda$ and $\Gamma$ are the local energy matrix and the $\mathcal{L}_{2}$ gains matrix defined in (12) and (13), respectively.

Proof: Let $x_{\mathrm{f} i}$ be the target state of the $i$-th cluster, and let $\left\|x_{\mathrm{f} i}\right\|_{2}=\alpha_{i}$. From equations (5) and (10), and from the definition of $\mathcal{L}_{2}$ gain [20] it follows that

$$
\left\|v_{i}\right\|_{2, T} \leq \frac{\alpha_{i}}{\lambda_{\min }^{1 / 2}\left(\mathcal{W}_{i, T}\right)}, \quad\left\|f_{i j}\right\|_{2, T} \leq \frac{\gamma_{i j} \alpha_{j}}{\lambda_{\min }^{1 / 2}\left(\mathcal{W}_{j, T}\right)}
$$


Moreover, due to the triangle inequality, we have

$$
\begin{aligned}
\left\|u_{i}\right\|_{2, T} & \leq\left\|v_{i}\right\|_{2, T}+\sum_{j \in \mathcal{N}_{i}}\left\|f_{i j}\right\|_{2, T} \\
& \leq \frac{\alpha_{i}}{\lambda_{\min }^{1 / 2}\left(\mathcal{W}_{i, T}\right)}+\sum_{j \in \mathcal{N}_{i}} \frac{\gamma_{i j} \alpha_{j}}{\lambda_{\min }^{1 / 2}\left(\mathcal{W}_{j, T}\right)}=\Gamma_{i} \Lambda^{1 / 2} \alpha
\end{aligned}
$$

where $\Gamma_{i}$ is the $i$-th row of $\Gamma$ defined in (13), and $\alpha$ is the vector of $\alpha_{i}$ with $i \in\{1, \ldots, N\}$. By using (13) and the fact that $\left\|u_{\mathcal{K}}^{\mathrm{d}}\right\|_{2, T}^{2}=\sum_{i=1}^{N}\left\|u_{i}\right\|_{2, T}^{2}$, we obtain

$\left\|u_{\mathcal{K}}^{\mathrm{d}}\right\|_{2, T}^{2} \leq \max _{\|\alpha\|=1} \alpha^{\top} \Lambda^{1 / 2} \Gamma^{\top} \Gamma \Lambda^{1 / 2} \alpha=\lambda_{\max }\left(\Lambda^{1 / 2} \Gamma^{\top} \Gamma \Lambda^{1 / 2}\right)$

from which the statement follows.

In Theorem 4.1 we derive a bound on the energy needed to control a network via our decoupled control law. Theorem 4.1 has several general consequences which we now describe. First, due to equation (5), if the set $\mathcal{K}$ of control nodes includes the boundary nodes of a network partition $\mathcal{P}$, then

$$
\lambda_{\min }\left(\mathcal{W}_{\mathcal{K}, T}\right) \geq \frac{1}{\left\|\Gamma \Lambda^{1 / 2}\right\|_{2}^{2}},
$$

where $\Lambda$ and $\Gamma$ are the local energy matrix and the $\mathcal{L}_{2}$ gains matrix for the partition $\mathcal{P}$. This bound on the smallest eigenvalue of the controllability Gramian is novel (see [21]), and it highlights that the controllability of a clustered network depends on the controllability of the isolated clusters via the matrix $\Lambda$, and on their interconnections strength via the $\mathcal{L}_{2}$ gains matrix $\Gamma$. Second, the control energy for our decoupled control law does not depend on the cardinality of the whole network. In fact, notice that

$$
\left\|\Gamma \Lambda^{1 / 2}\right\|_{2}^{2} \leq\|\Gamma\|_{2}^{2}\|\Lambda\|_{2} \leq\|\Gamma\|_{1}\|\Gamma\|_{\infty}\|\Lambda\|_{\infty},
$$

and that, independently of the network dimension, $\|\Gamma\|_{1}$ and $\|\Gamma\|_{\infty}$ remain bounded if, for instance, the network weights and the nodes degrees are bounded. A related example is in Section IV-C. Third and finally, since the energy to control a network via the decoupled control law depends on local properties of the network partitions, an appropriate partitioning method may be developed to optimize the performance of the decoupled control law. To this aim, we state the following corollary of Theorem 4.1, where we derive a bound on the control energy for our decoupled control law, which is proportional to the interconnection strength among clusters. Let $\Delta$ be the symmetric interconnection matrix defined by

$$
\Delta:=\left[\begin{array}{cccc}
1 & \left\|A_{12}\right\|_{2} & \cdots & \left\|A_{1 N}\right\|_{2} \\
\left\|A_{21}\right\|_{2} & 1 & \cdots & \left\|A_{2 N}\right\|_{2} \\
\vdots & \vdots & \ddots & \vdots \\
\left\|A_{N 1}\right\|_{2} & \left\|A_{N 2}\right\|_{2} & \cdots & 1
\end{array}\right] .
$$

Corollary 4.2: (Bound for network partitioning) Let $\gamma_{i j}$ be the $\mathcal{L}_{2}$ gain of the system $\left(A_{j}, B_{\mathcal{K}_{j}}, B_{\mathcal{K}_{i}}^{\top} A_{i j}\right)$, and let $\bar{\lambda}_{\max }=\max \left\{\lambda_{\max }\left(A_{i}\right): i \in\{1, \ldots, N\}\right\}<1$. Then,

$$
\gamma_{i j} \leq \frac{\left\|A_{i j}\right\|_{2}}{1-\bar{\lambda}_{\max }} \text {, for } j \in\{1, \ldots, N\} \backslash\{i\} \text {, }
$$

and, being $T$ the control horizon,

$$
\mathrm{E}\left(u_{\mathcal{K}}^{\mathrm{d}}, T\right) \leq \frac{\|\Lambda\|_{\infty}\|\Delta\|_{\infty}^{2}}{\left(1-\bar{\lambda}_{\max }\right)^{2}},
$$

where $\Lambda$ is the local energy matrix defined in equation (12), and $\Delta$ is the interconnection matrix defined in equation (17).

Proof: Recall that $\gamma_{i j}$ equals the $H_{\infty}$ gain of the transfer matrix of the system $\left(A_{j}, B_{\mathcal{K}_{j}}, B_{\mathcal{K}_{i}}^{\top} A_{i j}\right)$, that is,

$$
\gamma_{i j}:=\left\|B_{\mathcal{K}_{i}}^{\top} A_{i j}\left(z I-A_{j}\right)^{-1} B_{\mathcal{K}_{j}}\right\|_{H_{\infty}},
$$

where $\|\cdot\|_{H_{\infty}}$ denotes the $H_{\infty}$ norm [20]. Since the $H_{\infty}$ norm satisfies the submultiplicative property, we have

$$
\gamma_{i j} \leq\left\|B_{\mathcal{K}_{i}}^{\top}\right\|_{H_{\infty}}\left\|A_{i j}\right\|_{H_{\infty}}\left\|\left(z I-A_{j}\right)^{-1}\right\|_{H_{\infty}}\left\|B_{\mathcal{K}_{j}}\right\|_{H_{\infty}} .
$$

Notice that the $H_{\infty}$ norm of a constant transfer matrix coincides with its induced 2-norm. Finally we have $\left\|B_{\mathcal{K}_{i}}^{\top}\right\|_{2}=$ $\left\|B_{\mathcal{K}_{j}}\right\|_{2}=1$, and

$$
\begin{aligned}
& \left\|\left(z I-A_{j}\right)^{-1}\right\|_{H_{\infty}}:=\max _{\theta} \sigma_{\max }\left(\left(e^{-i \theta} I-A_{j}\right)^{-1}\right) \\
& =\max _{\theta}\left[\lambda_{\max }\left(\left(e^{i \theta} I-A_{j}\right)^{-1}\left(e^{-i \theta} I-A_{j}\right)^{-1}\right)\right]^{1 / 2} \\
& \left.=\max _{\theta}\left[\lambda_{\max }\left(I-2 \cos (\theta) A_{j}+A_{j}^{2}\right)^{-1}\right)\right]^{1 / 2} \\
& =\frac{1}{1-\lambda_{\max }\left(A_{j}\right)} \leq \frac{1}{1-\bar{\lambda}_{\max }},
\end{aligned}
$$

from which the first part of the statement follows. The second statement follows from (14), (16), and from the facts $\|\Gamma\|_{\infty}\left(1-\bar{\lambda}_{\max }\right) \leq\|\Delta\|_{\infty},\|\Gamma\|_{1}\left(1-\bar{\lambda}_{\max }\right) \leq\|\Delta\|_{1}=$ $\|\Delta\|_{\infty}$.

Analogously to equation (15), from Corollary 4.2 we conclude that, if the set $\mathcal{K}$ of control nodes includes the boundary nodes of a network partition $\mathcal{P}$, then

$$
\lambda_{\min }\left(\mathcal{W}_{\mathcal{K}, T}\right) \geq \frac{\left(1-\bar{\lambda}_{\max }\right)^{2}}{\|\Lambda\|_{\infty}\|\Delta\|_{\infty}^{2}},
$$

where $\Lambda$ and $\Delta$ are the local energy matrix and the interconnection matrix for the partition $\mathcal{P}$, respectively, and $\bar{\lambda}_{\text {max }}$ is a bound on the spectral radius of the clusters of $\mathcal{P}$.

We conclude this part by noting that our results lead to a novel notion of network controllability centrality, ${ }^{2}$ where network nodes are ranked according to the product of their local controllability degree and their interconnection strength with neighboring nodes. Our notion of network controllability centrality is motivated by Corollary 4.2, where the control energy is bounded by the scaled product of the worst-case control energy of the isolated clusters $\|\Lambda\|_{\infty}$ (least controllable cluster), and the worst-case clusters interconnection strength $\|\Delta\|_{\infty}$ (strongest interconnection strength). A comparison between controllability centrality and other centrality notions is left as the subject of future research.

\section{An example of network control via decoupled control law}

In this section we demonstrate our technique to control large networks with an example. Consider a circulant network $\mathcal{G}$ with $n=n_{\mathrm{b}} N$ nodes, $n_{\mathrm{b}}, N \in \mathbb{N}$, and adjacency

\footnotetext{
${ }^{2}$ Network centrality is a fundamental concept in network analysis [22].
} 


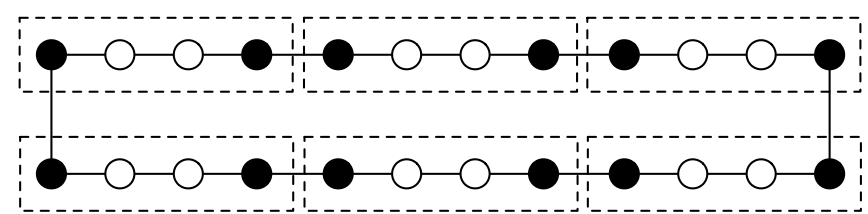

Fig. 2. A circulant network with $n=24$ nodes. The network is partitioned into $N=6$ clusters with $n_{\mathrm{b}}=4$ nodes each. Controlled nodes are in black.
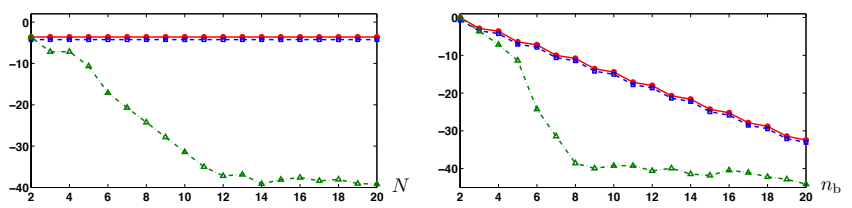

Fig. 3. In this figures we study circulant networks partitioned as in Section IV-C, and we compare (in a logarithmic scale) the performance of our decoupled control law against the minimum energy control law. In the left figure we maintain constant the number of nodes in each cluster, and we report as a function of the number clusters (see Section IV-C) (i) the smallest eigenvalue of the controllability Gramian with $T=\infty$ and boundary nodes as control nodes (solid red), (ii) the bound (15) for the energy performance (see Theorem 4.1) achieved by our decoupled control law (dashed blue), and (iii) the smallest eigenvalue of the controllability Gramian with $T=\infty$ and control nodes selected randomly (dashed-dotted green). Notice that the energy needed by our decoupled control law remains constant when the network cardinality grows (the number of control nodes grows as $2 \mathrm{~N}$ and that the number of nodes in each cluster remains constant). This property is not maintained if the control nodes are chosen randomly. In the right figure we report the same quantities as in the left figure, while maintaining constant the number of clusters and letting the number of nodes in each cluster grow. Notice that the smallest eigenvalue of the controllability Gramian and our bound (15) degrade with the same rate, while randomly selected control nodes require more energy.

matrix as in Example 3.1 with $\rho=0.5$. We partition $\mathcal{G}$ into $N$ clusters, so that each cluster contains $n_{\mathrm{b}}$ nodes. In particular, we label the nodes in increasing order, and for $i \in\{1, \ldots, N\}$ we define the $i$-th cluster to have vertices $\mathcal{V}_{i}:=\left\{(i-1) n_{\mathrm{b}}+1,(i-1) n_{\mathrm{b}}+2, \ldots, i n_{\mathrm{b}}\right\}$ and control nodes $\mathcal{K}_{i}:=\left\{(i-1) n_{\mathrm{b}}+1, i n_{\mathrm{b}}\right\}$.

See Fig. 2 for an example with $n_{\mathrm{b}}=4$ and $N=6$. It can be numerically verified ${ }^{3}$ that the set $\mathcal{K}$ of control nodes is optimal, in the sense that it solves the maximization problem

$$
\begin{array}{cl}
\max _{\mathcal{K} \subseteq\{1, \ldots, n\}} & \lambda_{\min }\left(\mathcal{W}_{\mathcal{K}, \infty}\right), \\
\text { subject to } & |\mathcal{K}|=2 N .
\end{array}
$$

In Fig. 3 we validate Theorem 4.1 and equation (15). Notice that, although conservative, our bound (15) captures the fact that circulant networks can be driven with constant energy to any (unit norm) target state independently of the network dimension; this result is compatible with our analysis in Theorem 3.1 and in Section IV-B. Moreover, our decoupled control law is a distributed control law achieving this performance. Finally, it can be shown that for circulant networks, and in fact for all $d$-dimensional torus networks, the diagonal entries of $\left(I-A A^{\top}\right)^{-1}$ are all equal to each other. Thus, the selection of the control nodes for the maximization of the trace of the Gramian is in this case equivalent to a random positioning of the control nodes [1].

\footnotetext{
${ }^{3}$ Due to computational complexity, we have solved the maximization problem (18) for the cases $n_{\mathrm{b}}=4$ and $N \in\{2, \ldots, 6\}$.
}

\section{CONClusion}

In this work we study the problem of controlling complex networks to a target state. We adopt the smallest eigenvalue of the controllability Gramian, which quantifies the worstcase control energy, as measure of network controllability. First, we characterize tradeoffs between the number of control nodes and the control energy as a function of the network dynamics. Then, we develop a control strategy with performance guarantees, consisting of a method to select control nodes based on network partitioning, and a distributed control law to drive the network to the target state.

\section{REFERENCES}

[1] F. Pasqualetti, S. Zampieri, and F. Bullo, "Controllability metrics, limitations and algorithms for complex networks," IEEE Transactions on Control of Network Systems, Sep. 2013, to appear.

[2] Y. Y. Liu, J. J. Slotine, and A. L. Barabási, "Controllability of complex networks," Nature, vol. 473, no. 7346, pp. 167-173, 2011.

[3] K. J. Reinschke, Multivariable Control: A Graph-Theoretic Approach. Springer, 1988.

[4] W. M. Wonham, Linear Multivariable Control: A Geometric Approach, 3rd ed. Springer, 1985.

[5] G. Parlangeli and G. Notarstefano, "On the reachability and observability of path and cycle graphs," IEEE Transactions on Automatic Control, vol. 57, no. 3, pp. 743-748, 2012.

[6] Y.-Y. Liu, J.-J. Slotine, and A.-L. Barabási, "Observability of complex systems," Proceedings of the National Academy of Sciences, vol. 110, no. 7, pp. 2460-2465, 2013.

[7] J. M. Dion, C. Commault, and J. van der Woude, "Generic properties and control of linear structured systems: a survey," Automatica, vol. 39, no. 7, pp. 1125-1144, 2003.

[8] F.-J. Müller and A. Sachuppert, "Few inputs can reprogram biological networks," Nature, vol. 478, no. 7369, pp. E4-E4, 2011.

[9] N. J. Cowan, E. J. Chastain, D. A. Vilhena, J. S. Freudenberg, and C. T. Bergstrom, "Nodal dynamics, not degree distributions, determine the structural controllability of complex networks," PLoS ONE, vol. 7, no. 6, p. e38398, 2012.

[10] R. E. Kalman, Y. C. Ho, and S. K. Narendra, "Controllability of linear dynamical systems," Contributions to Differential Equations, vol. 1, no. 2, pp. 189-213, 1963.

[11] A. Rahmani, M. Ji, M. Mesbahi, and M. Egerstedt, "Controllability of multi-agent systems from a graph-theoretic perspective," SIAM Journal on Control and Optimization, vol. 48, no. 1, pp. 162-186, 2009.

[12] A. Olshevsky, "The minimal controllability problem," 2013, arXiv preprint arXiv:1304.3071.

[13] G. Notarstefano and G. Parlangeli, "Controllability and observability of grid graphs via reduction and symmetries," IEEE Transactions on Automatic Control, vol. 58, no. 7, pp. 1719-1731, 2013.

[14] J. Sun and A. E. Motter, "Controllability transition and nonlocality in network control," Physical Review Letters, vol. 110, no. 20, p. 208701, 2013.

[15] A. C. Antoulas, D. C. Sorensen, and Y. Zhou, "On the decay rate of Hankel singular values and related issues," Systems \& Control Letters, vol. 46, no. 5, pp. 323-342, 2002.

[16] T. Kailath, Linear Systems. Prentice-Hall, 1980.

[17] R. W. Brockett, Finite Dimensional Linear Systems. Wiley and Sons, 1970.

[18] F. L. Bauer and C. T. Fike, "Norms and exclusion theorems," $\mathrm{Nu}$ merische Mathematik, vol. 2, no. 1, pp. 137-141, 1960.

[19] S. Fortunato, "Community detection in graphs," Physics Reports, vol. 486, no. 3-5, pp. 75-174, 2010.

[20] S. Skogestad and I. Postlethwaite, Multivariable Feedback Control Analysis and Design, 2nd ed. Wiley, 2005.

[21] W. H. Kwon, Y. S. Moon, and S. C. Ahn, "Bounds in algebraic Riccati and Lyapunov equations: a survey and some new results," International Journal of Control, vol. 64, no. 3, pp. 377-389, 1996.

[22] M. E. J. Newman, Networks: An Introduction. Oxford University Press, 2010. 